\title{
LA RECEPCIÓN DE LA DOCTRINA ARISTOTÉLICA DEL INTELECTO EN AL-FÂRÂBÎ Y EL PROBLEMA DE LA INMORTALIDAD DEL ALMA HUMANA
}

\author{
THE RECEPTION OF THE ARISTOTELIAN DOCTRINE OF \\ THE INTELLECT IN AL-FÂRÂBÎ AND THE PROBLEM OF THE \\ IMMORTALITY OF THE HUMAN SOUL
}

\author{
Matías Leiva \\ Universidad de los Andes \\ Monseñor Álvaro del Portillo 12455 \\ Las Condes, Santiago \\ maleiva1@miuandes.cl
}

\begin{abstract}
Resumen
La filosofía árabe clásica constituye un ejemplo claro de la recepción que tuvieron las ideas de la filosofía griega en el mundo oriental. En este artículo abordaremos la recepción del pensamiento aristotélico en al-Fârâbî, uno de los más ilustres filósofos árabes. Intentaremos demostrar tres puntos: primero, que el problema de la inmortalidad del alma humana individual en Aristóteles constituye una aporía; segundo, que la solución ofrecida por al-Fârâbî contiene elementos que se alejan de lo expuesto por Aristóteles y que tienen un claro eco neoplatónico y, tercero, que la filosofía farabiana es una síntesis de Platón y Aristóteles.
\end{abstract}


Para ello, revisaremos brevemente la caracterización que hace el filósofo griego del intelecto en el libro III del De anima y la recepción que tienen estas ideas en el pensamiento de al-Fârâbî y que, a su vez, le permiten afirmar la inmortalidad del alma.

Palabras clave: alma, intelecto, inmortalidad, separabilidad, neoplatonismo

\section{Abstract}

Classic Arabic philosophy constitutes a clear example of the reception of classical Greek philosophy in the eastern world. In this article we examine the reception of the Aristotelian thinking in al-Fârâbî, one of the most remarkable Arab philosophers. We prove three points: first, that the problem of the immortality of the individual human soul constitutes an aporia in Aristotle; second, that the solution offered by al-Fârâbî contains elements that are far from what Aristotle stated and have a clear Neoplatonic echo; and third, that Farabian philosophy constitutes a synthesis between Aristotle and Plato. To achieve this, we will briefly review the characterization of the intellect the Greek philosopher makes in Book III of the De Anima and the reception these ideas had in al-Fârâbî's philosophy which, in turn, allowed him to state the immortality of the soul.

Keywords: Soul, Intellect, Immortality, Separability, Neoplatonism 


\section{El PROBlema de la inMortalidad del ALMA EN LOS TEXTOS ARISTOTÉLICOS}

Los escritos filosóficos, científicos y metafísicos de Aristóteles han suscitado siglos y siglos de discusión y estudio. Uno de sus textos de madurez ${ }^{1}$, el De anima ${ }^{2}$, tiene como preocupación central el alma y aborda diversos e interesantes problemas: la relación del cuerpo y el alma, el estatuto ontológico del alma, sus tipos, sus facultades y, finalmente, la importancia del intelecto como facultad superior en la jerarquía de las almas. Tradicionalmente se le considera un escrito de madurez pues incorpora elementos ya abordados en otras obras, especialmente en la Metafísica, sus textos sobre ética y sus obras biológicas. Uno de los problemas que aborda, pero no directamente, es la inmortalidad del alma humana. Si bien cuando se trata de las almas vegetal y animal no hay indicio alguno que sugiera la posibilidad de considerarlas como inmortales ${ }^{3}$, cuando habla del alma humana, asociada estrechamente al intelecto, deja ciertas puertas abiertas que podrían llevarnos a pensar que entretuvo la idea de que, gracias a dicha facultad, el alma intelectiva extenderían su existencia luego de la corrupción corporal. Dicho lo anterior, es menester reconocer que el problema está lejos de estar cerrado. A los largo de los siglos el De anima ha sido utilizado para afirmar y negar la inmortalidad del alma humana.

En este primer apartado revisaremos los textos que nos ofrece Aristóteles en el De Anima para acercarnos al problema de la inmortalidad. Aunque podrían citarse y analizarse múltiples pasajes, hay dos que son

$1 \quad$ El problema de la cronología de los textos de Aristóteles y el lugar que ocupa el De anima en el sistema aristotélico ha generado un renovado interés desde el siglo pasado hasta hoy. Para hacerse una idea general de los inicios de este problema se sugiere revisitar la discusión de Jaeger y Nuyens. Para nuestros efectos, arrancaremos desde una idea ampliamente aceptada, a saber, que el De anima constituye un escrito aristotélico de madurez, sin entrar en la discusión detallada del orden cronológico del corpus aristotélico.

2 Para la citación de los textos clásicos se utilizará el formato estandarizado de Oxford, disponible en https://oxfordre.com/classics/page/ocdabbreviations/abbreviations.

3 La tradicional clasificación de los tipos de alma se puede observar en De anima, capítulos 1 y 2 . 
particularmente representativos de la relación cuerpo-alma y su posible inmortalidad y que originan el problema que aborda el neoplatonismo y, posteriormente, la filosofía árabe. El primero se encuentra en los párrafos finales de II.2:

Pues "sustancia" se dice de tres maneras, según dijimos: forma, materia, y lo que se compone de ambas. Y de ellos, la materia es la potencia y la forma actualidad; dado que lo que se compone de ambas cosas es animado, el cuerpo no es la actualidad del alma, sino que esta [es la actualidad] de un cierto cuerpo. Es también por esa razón que hacen una suposición apropiada aquellos a los que les parece que el alma no existe sin un cuerpo ni es un cierto cuerpo. No es como [pensaron] los que nos precedieron, que la adjuntaban a un cuerpo, sin determinar para nada en qué o en cuál [cuerpo] [...] Es así, como sucede también según la definición, pues la actualidad de cada cosa sobreviene por naturaleza a lo que existe en potencia y a la materia apropiada. Por consiguiente, que [el alma] es una cierta actualidad, es decir, un principio explicativo de lo que tiene potencia de ser tal, es evidente a partir de estos [argumentos]. (De an. 414a, 15-30)

A partir de este texto podemos extraer algunas conclusiones relevantes para comprender la relación entre cuerpo y alma y el estatuto que Aristóteles le confiere al alma. En primer lugar, el filósofo alude a su conocida teoría hilemórfica para situar al cuerpo y al alma dentro de ese marco de referencia ${ }^{4}$. El cuerpo corresponde a la materia y el alma a la forma. Así, el alma es asociada con el acto y se constituye como la actualidad del cuerpo (entelequia), quedando el cuerpo relegado a la mera potencialidad. De aquí podemos inferir, además, que el alma es de carácter inmaterial. Dado que las formas son inmateriales, y el alma es la forma del cuerpo, el alma debe ser inmaterial. Podríamos asumir

Como es sabido, la teoría hilemórfica es expuesta en la Metafísica. Para un tratamiento problematizado de ella, revisar el tradicional libro zeta de la obra, especialmente el rol que juega cada uno de estos principios (materia, forma y compuesto) en relación con la sustancia. 
apresuradamente que, en este pasaje, se nos presenta el alma como inmortal, basándonos en la incorruptibilidad de lo inmaterial. Sin embargo, la conclusión que ofrece Aristóteles en las líneas siguientes se dirige en la dirección contraria: "el alma no existe sin un cuerpo". A pesar de que el alma es inmaterial y que es en acto, no puede existir sin la materia. Esta afirmación pareciera reforzar la teoría hilemórfica que está a la base: la sustancia se constituye de los dos principios y no es posible la existencia del uno sin el otro (en las sustancias compuestas, como es el caso del ser humano). Es impensable una materia que no tenga una forma, y una forma sustancial que no se realice en una materia específica. Por otra parte, se podría insistir en el argumento inmortalista y buscar razones en otros pasajes para afirmar que hay formas que existen sin materia. Sin embargo, ya que la afirmación de Aristóteles es tan definitiva, perseguir esa línea argumentativa sería un ejercicio hermenéutico poco fiel que se aleja de la fuente. Posteriormente, se refiere a la originalidad y contexto de su propuesta: "No es como [pensaron] los que nos precedieron, que la adjuntaban a un cuerpo". Podría estarse refiriendo a una cierta concepción dualista, donde se entiende al ser humano como un alma a la que se le añade un cuerpo, accidentalmente si se quiere. Quizás alude a Platón que debía ya estar muerto, pues el De anima corresponde a la madurez filosófica de Aristóteles. Podría estar discutiendo también con los discípulos de su maestro, o con alguna corriente presocrática de corte dualista $^{5}$. En cualquier caso, el estagirita refuerza la idea de la unidad substancial del cuerpo y el alma (Guerrero 46) y señala explícitamente que el alma no puede vivir sin el cuerpo, rechaza el dualismo y niega la inmortalidad del alma. Afirmar tal inmortalidad sería pensar como "los que nos precedieron", es decir, equivocadamente. A partir de aquí,

Esta posibilidad, sin embargo, es la menos probable. Como es sabido, Aristóteles dedica el libro I completo a llevar a cabo un ejercicio dialéctico con los filósofos presocráticos, señalando sus fortalezas y debilidades. Algunas de las propiedades que ellos atribuyeron al alma son, finalmente, recogidas por Aristóteles (la inmaterialidad, por ejemplo) y otras son descartadas por considerarlas incongruentes con el marco metafísico general que está a la base de su psicología (el alma como una armonía, por ejemplo). 
entonces, afirmar la inmortalidad del alma sería un ejercicio forzado y poco fiel a lo expuesto en este pasaje de la obra.

Pasemos a revisar el otro pasaje clave para la discusión acerca de la inmortalidad del alma, que es recogido por los neoplatónicos y, a través de ellos, por el mundo árabe 6 . Este se encuentra en III.5:

Puesto que en la naturaleza toda hay algo que, en un sentido, es materia para cada tipo de cosa -y eso es lo que en potencia es todas aquellas cosas-, y otro sentido, es la causa, es decir el agente, por producir todas las cosas -como el arte respecto de la materia-, es necesario que estas distinciones se den también en el alma. Y también hay un intelecto que es tal por llegar a ser todas las cosas, y otro por producirlas todas, que es como un cierto tipo de estado, como la luz [...] También este intelecto es separable e impasible y sin mezcla por cuanto sustancialmente es en acto [...] Una vez separado, sin embargo, eso solo es lo que precisamente es, y eso solo es inmortal y eterno; no obstante, no [lo] recordamos, porque eso es impasible, pero el intelecto paciente es corruptible y sin eso [uno] nada intelige. (De an. 430a, 10-26)

Este pasaje ha generado siglos y siglos de discusión antropológica, epistemológica e incluso cosmológica. Es desde aquí que la filosofía neoplatónica afirma la posibilidad de la inmortalidad del alma en la psicología de Aristóteles y desde donde nacen las distinciones en las facultades humanas que permitirán que diversos filósofos construyan sus propias teorías sobre el asunto, llegando incluso hasta la escolástica, diecisiete siglos después. Revisemos, brevemente, los elementos centrales que lo componen. El filósofo comienza señalando que existen dos tipos de intelectos en el alma humana: por una parte está el intelecto paciente ${ }^{7}$, capaz de hacerse todas las cosas, es decir, de recibir las formas o esencias y, por otra, el intelecto

6 Si bien no es el único pasaje que ofrece problemas y que puede haber sido recogido por el neoplatonismo, es el más representativo. En cualquier caso, en general el libro III plantea una serie de problemas respecto de la comprensión tradicional del intelecto.

7 El calificativo de paciente (pathetikós) es acuñado por Alejandro de Afrodisias. Si bien Aristóteles es el autor de la distinción entre los dos intelectos, solamente denominó al agente. El intelecto paciente recibe únicamente una caracterización en el De anima. 
agente, que es capaz de hacer todas las cosas, a saber, formar conceptos universales (noêmata) a partir de ese conocimiento de las esencias. El intelecto paciente está asociado con la potencia y el agente con el acto. El punto más polémico es donde se describe el intelecto agente como separable, inmortal y eterno. Lamentablemente el filósofo no nos provee de un marco interpretativo desde el cual comprender estos atributos del alma. Por la fuerza del lenguaje y la resonancia que tiene con la Metafísica XII algunos autores han sugerido que se refiere al intelecto divino. Sin embargo, dado que esta caracterización se encuentra seguida de las distinciones sobre el intelecto humano, es más plausible pensar que se está refiriendo al segundo y no al primero. Sobre la eternidad y la inmortalidad, no hay otros pasajes que lo expliquen ni que ofrezcan claves interpretativas que guíen una exégesis rigurosa. El carácter de separable (chôristón), por su parte, aparece ya en la Metafisica, especialmente en el libro zeta y en el comienzo del De anima ${ }^{8}$. En este último se hace la distinción entre la separación teórica o conceptual y la separación material o topológica (I.1). Sin embargo, como el libro I del De anima es, fundamentalmente, una revisión dialéctica del alcance y objetivos del tópico a mano, así como de las opiniones que hay al respecto de tal o cual tema específico, Aristóteles no se pronuncia allí por el sentido de esa separabilidad. Lamentablemente, no lo hace en el resto de la obra tampoco.

¿Qué quiere decir Aristóteles cuando se refiere al intelecto agente como algo separable? Algunos han sostenido otras teorías; por ejemplo, que esa separabilidad no es más que una distinción entre la operación del intelecto agente respecto del paciente, otros, que se trata simplemente de la aserción de que el intelecto opera desde el alma y no requiere de un órgano específico (Zingano 153; 176-7). Finalmente, hay una larga tradición neoplatónica que ve en este pasaje una afirmación clara y definitiva de la inmortalidad del alma. Las soluciones son múltiples, pero nos centraremos en la que ofrece la visión neoplatónica oriental, que juega un rol clave para

8 El problema de la separabilidad en la Metafísica está presente desde el inicio de la obra. Para un tratamiento de este concepto y las diferencias entre Aristóteles y su maestro, ver Di Camillo. 
la postura que asume la filosofía árabe sobre el problema de la inmortalidad del alma. En el siguiente apartado nos referiremos a la recepción que tuvo esta doctrina del intelecto en la filosofía de al-Fârâbî y su propuesta para resolver esta aporía.

\section{LA RECEPCIÓN DE LA DOCTRINA ARISTOTÉLICA DEL INTELECTO EN AL-FÂRÂBî Y LA INMORTALIDAD DEL ALMA}

En el mundo árabe el texto fundamental y fuente de conocimiento verdadero es el Corán. Sin embargo, tal como sucede con la Biblia en el mundo cristiano occidental, este libro sagrado no opera como un tratado científico del cual emanan todas y cada una de las verdades que responden a las infinitas preguntas que surgen de la curiosidad humana. $\mathrm{Al}$ ser una verdad revelada, aunque permite la búsqueda de otras verdades que expliquen, fundamenten y profundicen dichas preguntas, no agota el conocimiento técnico y científico que se puede tener de la realidad. Este es uno de los factores clave que dieron origen a la filosofía clásica árabe. La mezcla entre las nacientes investigaciones y planteamientos intelectuales, el contacto cultural con otras cosmovisiones existentes y la incapacidad de la verdad revelada para responder a los aspectos más específicos de la realidad, llevaron al mundo árabe a recurrir a la filosofía para intentar abordar y responder sus preguntas (Guerrero 11-36). Y acudieron a los más elevados representantes de aquella disciplina hasta el momento, es decir, la filosofía griega, particularmente Aristóteles, Platón y sus seguidores neoplatónicos (Guerrero 77). El punto de inicio del influjo del mundo griego en la cultura árabe se ha situado tradicionalmente a fines del siglo VIII, a partir de ciertas traducciones que surgen y circulan en Oriente. Sin embargo, diversos autores han sugerido que la entrada de las cosmovisiones griegas puede haberse producido antes (Thillet 60) ${ }^{9}$. En cualquier caso, la recepción de esta filosofía occidental permitió la formación de lo que hoy

$9 \quad$ Gilson sugiere que el mundo griego penetró en oriente incluso antes del cierre de la Escuela de Alejandría, a través de Siria (La filosofía 321-2). 
conocemos como filosofía árabe o islámica (Guerrero 78-84). A su vez, las reflexiones del mundo oriental permitieron que la filosofía medieval, y la escolástica en particular, alcanzara el grado de profundidad y trascendencia que goza hasta el día de hoy (López-Farjeat, "La inmortalidad..." 577). Así, entender esa filosofía árabe como una mera extensión de la filosofía clásica griega es un error. Bajo la profunda convicción de que las filosofías de Platón y Aristóteles eran compatibles, estos filósofos intentaron explicarlas y sintetizarlas. Llevando a cabo esa ambiciosa empresa, como veremos, lograron darle una impronta única a su filosofía, dejando su huella propia en la historia del pensamiento (Gilson, La filosofía 324).

Del encuentro cultural entre oriente y occidente, la filosofía aristotélica fue la que recibió la mayor atención ${ }^{10}$. De todo el trabajo filosófico de Aristóteles lo primero en ser estudiado, en línea con las disciplinas emanadas del estudio del Corán, fueron los textos lógicos, por los que los cristianos siriacos tenían una especial predilección (López-Farjeat, "La inmortalidad..." 580-1). A medida que se fueron traduciendo otros textos aristotélicos, comenzaron a estudiarse y a producirse múltiples paráfrasis, poniendo especial atención en el texto que nos interesa en este trabajo, el De anima. El estagirita fue reverenciado como un gran maestro de filosofía y se escribieron múltiples biografías sobre él (Guerrero 85-6). Grandes filósofos, Averroes entre ellos, no escatimaron en calificativos para elogiarlo ${ }^{11}$. Sin embargo, es clave considerar que la filosofía peripatética que recibió el mundo árabe tenía un fuerte componente neoplatónico (Gilson 322). Esto significó, entre otras cosas, que buena parte de las reflexiones filosóficas, especialmente la cosmología y metafísica que al-Fârâbî heredó eran, en realidad, la filosofía de Platón y sus seguidores (Gilson, La filosofía 322). Como veremos más adelante, esto jugó un rol clave en el problema de la inmortalidad del alma. Así, la influencia platónica en la filosofía árabe, siendo explícita o no, fue un elemento crucial en su conformación y contenido ${ }^{12}$. El influjo

10 Para un breve recorrido cronológico de la penetración de Aristóteles en Oriente, ver López-Farjeat "La inmortalidad del alma" 578-83 y Guerrero 85-92.

11 Para los múltiples elogios de Averroes hacia Aristóteles ver Guerrero 85-6.

12 Diversos autores han trabajado este tema. Ver por ejemplo Galston, Mahdi y Rosenthal. 
platónico fue especialmente útil en tanto permitió enriquecer la filosofía aristotélica en aquellos aspectos en que no se acomodaban a la visión árabe del mundo, integrando elementos propiamente platónicos a las aporías de la filosofía de Aristóteles, lo que le permitió a los filósofos árabes salvar diversos problemas (Guerrero 113-4). Así, los pensadores clásicos, al-Fârâbî entre ellos, utilizaron una filosofía aristotélica neoplatonizada para resolver los problemas intelectuales propios del islam (Guerrero 161).

Nuestro autor de cabecera, Abû Nașr Muḥammad ibn al-Faraj alFârâbî, fue uno de los grandes filósofos, sino el más grande, del período árabe clásico (Guerrero 153-4). Convencido de que la filosofía griega era compatible con la revelación del islam, produjo una filosofía que, aunque poco estudiada en el mundo occidental, resultó clave para el desarrollo del pensamiento medieval cristiano y su recepción de la filosofía peripatética. En él se puede apreciar no solo un manejo excepcional de la tradición griega clásica, sino también una clara asimilación e incorporación de sus ideas (Guerrero 158-9). Semejante a Platón, y recogiendo muchos de sus planteamientos, la preocupación principal de al-Fârâbî estaba relacionada con las condiciones requeridas para que el ser humano alcanzara el conocimiento verdadero y, de esa forma, la felicidad. Su filosofía, por tanto, ya sea política, metafísica o psicológica, debe ser comprendida bajo ese prisma, es decir, como un esfuerzo en la búsqueda de la perfección humana (Guerrero 161).

Del conjunto de la filosofía de Aristóteles, como hemos señalado, el texto que recibió más atención en el mundo árabe, que tuvo mayor influencia y, aparentemente, gozó de mayor cantidad de traducciones y versiones fue el De anima (López-Farjeat, "La inmortalidad..." 581) ${ }^{13}$, iniciándose su estudio, probablemente, con al-Kindî ${ }^{14}$. Junto con esta obra, es muy probable que hayan recibido, además, los comentarios de Temistio, Olimpiodoro, Simplicio y Alejandro de Afrodisias (López-Farjeat, "La inmortalidad..." 581-2). Para el siglo X el De anima ya contaba con una

\footnotetext{
13 Para una historia de las traducciones y versiones que se transmitieron del De anima, ver Guerrero 91-110.

14 Guerrero dedica un capítulo completo de su libro a revisar la recepción de la psicología aristotélica en al-Kindî (111-49).
} 
o varias traducciones maduras y trabajadas al siríaco, lo que fomentó su estudio y paráfrasis (Guerrero 92) ${ }^{15}$. Fue ampliamente conocido y trabajado por al-Kindî y al-Fârâbî y se erigió como uno de los textos predilectos de los círculos filosóficos de Bagdad durante ese siglo. Comentado y elogiado por Averroes, fue también conocido y estudiado por Avempace (Guerrero 109). Sin embargo, como mencionamos, la comprensión que tuvo el mundo árabe del De anima estuvo fuertemente marcada por elementos neoplatónicos.

El primer problema que debemos abordar para acercarnos al tópico de la inmortalidad del alma en al-Fârâbî es la fuente desde la cual arranca. El principal texto que le permite al filósofo árabe articular su teoría es la Theologia de Aristóteles (López-Farjeat, "La inmortalidad..." 578). Sin embargo, como sabemos, el estagirita no escribió nunca una obra con ese título, por lo tanto, sabemos que la fuente del filósofo árabe es un texto apócrifo, atribuido a Aristóteles, pero no de su autoría. Al revisar el texto, el lector puede adverti que la Theologia es, en realidad, las Enéadas de Plotino con añadiduras, comentarios e inserciones. Más específicamente, se trata de las Enéadas IV, V y VI ${ }^{16}$. En la actualidad la Theologia se entiende como parte del corpus del Plotino árabe, por lo que su análisis debe llevarse a cabo desde esa mirada ${ }^{17}$. Fue probablemente a través de Plotino, difundido por Porfirio, que las ideas psicológicas de Aristóteles terminaron de teñirse de platonismo e hicieron su entrada al mundo oriental ${ }^{18}$, siendo muy influyentes en al-Kindî, al-Fârâbî y Ibn Sinâ (López-Farjeat, "La inmortalidad..." 578).

Antes de introducirnos en la Theologia es importante considerar dos puntos fundamentales. El primero dice relación con la doctrina del intelecto de Aristóteles, fundamentalmente con el pasaje revisado más arriba (De an.

15 Peters realiza un interesante estudio sobre las traducciones que se llevaron a cabo de Aristóteles y sus comentarios. En esta línea ver también Guerrero (92-110). Para una discusión moderna sobre las primeras traducciones del De Anima, ver Minio-Paluello.

16 Cruz Hernández explica la estructura de la Theologia y como se construye a partir de las Enéadas y las diversas inserciones que tiene el texto (87-110). En esta línea, ver también Adamson, "The Theology..." 1.

17 Para un interesante estudio sobre el origen y la historia de la Theologia, ver Zimmermann. Por cuestiones de espacio no abordaremos esta temática aquí.

18 Para el fundamental rol que jugó Porfirio en la construcción y edición del texto, ver Cruz Hernández (87-110) y Zimmermann. 
III, 5.430a, 10-26), y más específicamente al problema de la separabilidad del intelecto. Como dijimos, esta idea ha atravesado siglos de estudios y diversos filósofos han intentado comprenderla en el marco del corpus aristotélico. Uno de los primeros en llevar a cabo esta tarea y que, a juicio de Dieterici, marcó el rumbo de las interpretaciones venideras, incluyendo la de al-Fârâbî, fue Alejandro de Afrodisias (Dieterici XVI). Algunas décadas más tarde, Gilson reafirmó esta idea, señalando que Alejandro fue la fuente por excelencia para las doctrinas del intelecto en el mundo árabe (Gilson, "Les sources gréco-arabes..." 7 y La filosofía 323-4) ${ }^{19}$. Este filósofo neoplatónico fue pionero en intentar sintetizar completamente a Platón y Aristóteles, mezclando elementos de ambos y resolviendo, desde su perspectiva, la ambigua doctrina aristotélica del intelecto (Guerrero 63). En el pasaje revisado del De anima vemos que para Aristóteles el intelecto agente forma parte de las potencias del ser humano, y es gracias a él que logra conocer verdaderamente; opera en conjunto con el intelecto paciente o potencial y se le relaciona de forma primordial con el acto. Alejandro, con su veta platonizante, comprende el intelecto humano de una forma distinta, como pura potencia, como mera capacidad para ser impresionado, recibir las formas y asemejarse a los inteligibles (Guerrero 63). Entendiéndolo así, relega toda actualidad del intelecto humano. De esa forma, y dado que el intelecto agente es puro acto, entonces no puede ser parte del intelecto propiamente humano, es decir, una facultad humana, debe ser algo distinto, separado del ser humano. La ambigüedad de la afirmación de Aristóteles en el pasaje De anima, permite a Alejandro tomar el intelecto agente y separarlo de la naturaleza humana y ponerlo en un plano superior de la realidad o, en términos platónicos, en el plano de las ideas ${ }^{20}$.

El segundo punto dice relación con la recepción de esta idea en la filosofía árabe. Como ya mencionamos, si la entrada al mundo oriental de la psicología aristotélica fue Plotino, y este recibió de Alejandro la síntesis

\footnotetext{
19 Existen algunos autores que niegan esta influencia. Ver, por ejemplo, Guerrero 61-3.

20 En Teofrasto hay un pensamiento intermedio. Para él, si bien el alma viene desde fuera (por tanto, es separada) se hace parte del ser humano de forma natural al momento de la generación (Guerrero 60-1).
} 
aristotélica-platónica, es natural que en al-Fârâbî encontremos una idea similar a la expuesta por él. Por ello, en la cosmología farabiana se puede ver claramente la influencia de Alejandro de Afrodisias y su entendimiento del intelecto agente. En La ciudad ideal al-Fârâbî expone su cosmología, es decir, la manera en que está compuesta y estructurada la realidad y la jerarquía que tienen los principios que la conforman ${ }^{21}$. Mencionaremos brevemente esa jerarquía, que sigue un modelo emanacionista, para entender la manera en que concibe que el intelecto agente sea separado. En primer lugar está el Ser Primero, Primer Existente o Primera Causa. Es inmaterial, uno, separado, no admite multiplicidad y todo lo existente proviene de él. Luego están los intelectos segundos, también incorpóreos y separados; proceden del Primer Existente y son la causa de los cuerpos celestes. Existen nueve esferas celestes, y un intelecto para cada una. Pero hay otro intelecto que actúa, sin corresponder a una esfera celeste, al igual que ellas, de manera directa sobre el mundo sublunar. Recibe el nombre de intelecto agente, y como intelecto segundo, es también inmaterial e incorpóreo. A partir de él existen las almas sublunares y los cuatro elementos. Además, es "el proveedor de los primeros principios del entendimiento, a través de los cuales los seres humanos pueden alcanzar la felicidad" (López-Farjeat, "Cosmología..." 17). Estos tres primeros principios no son cuerpos ni interactúan con los cuerpos. Los tres segundos, siendo también incorpóreos, sí tienen relación y actúan sobre los cuerpos. Siguiendo la nomenclatura aristotélica, al-Fârâbî los denomina alma, forma y materia respectivamente ${ }^{22}$. A partir de aquí vemos la deuda con Alejandro de Afrodisias y cómo en la jerarquía de los seres propuesta por al-Fârâbî, el intelecto agente queda relegado de la realidad sublunar o mundana como lo habría concebido Aristóteles; más bien se trata de un principio separado del mundo y, específicamente, de la realidad del ser humano. Aquí se juega la importancia de la interpretación

21 Para la cosmología y la jerarquía ontológica ver al-Fârâbî, La ciudad ideal, especialmente los primeros capítulos (I-XIII) y el "Libro de la política" 53-68. Resultan también iluminadoras las exposiciones de López-Farjeat “Cosmología” 16-24 y Guerrero 161-70.

22 Los problemas interpretativos y exegéticos que plantean estos principios son diversos y no atañen directamente al tema de este artículo, razón por la cual no nos detendremos en su explicación. 
de esa separabilidad. Es a partir de esta pequeña, pero muy significativa distinción, más ciertas consideraciones sobre la relación cuerpo-alma, que se estructura la teoría de la inmortalidad del alma en el neoplatonismo y en al-Fârâbî.

De los tres últimos principios (alma, forma y materia) nos ocuparemos únicamente del alma y mencionaremos brevemente su origen ${ }^{23}$. Al-Fârâbî sigue los textos biológicos de Aristóteles y señala que el alma, siendo forma, es inmaterial y viene dada por el macho. A su vez, el cuerpo es material y viene dado por la hembra ${ }^{24}$. Al-Fârâbî, recogiendo el conocimiento científico del estagirita, detalla el proceso biológico mediante el cual el semen (que aporta la forma) se une con la sangre del útero (que aporta la materia) y dan lugar al embrión (López-Farjeat, "Cosmología” 19-20). A medida que las distintas partes del cuerpo se van desarrollando, el ser vivo va adquiriendo las facultades que le corresponden según su nivel de vida (nutritiva, perceptiva y racional). Cuando se trata de la vida racional, el ser humano se eleva por sobre los otros seres en tanto su facultad es más perfecta y le permite el conocimiento, caracterizado por la adquisición de los inteligibles. Esa alma humana, dirá al-Fârâbî, es capaz de alcanzar tal estado de perfección, que ya no requerirá del cuerpo para existir (al-Fârâbî, Obras filosóficas 62-3) o, dicho de otra forma, existir separado de la materia. Como veremos, el rol del intelecto agente, que no forma parte de ese ser humano, sino que es separado, es crucial para lograr la existencia más allá de la realidad material somática.

Mencionamos anteriormente que el texto por excelencia desde el cual al-Fârâbî articula su teoría de la inmortalidad del alma es la Theologia. Para comprender dicha articulación, revisaremos algunos pasajes a la luz de las Enéadas de Plotino y del De anima de Aristóteles ${ }^{25}$. Sin embargo, es importante señalar que al-Fârâbî no cita ni menciona la Theologia en el

23 Para el origen del alma ver López-Farjeat, "Cosmología...” 18-21.

24 Para el proceso de origen del cuerpo y el alma ver al-Fârâbî, On the perfect State 186-97. Parte de esta visión se puede rastrear hasta Aristóteles: Gen. an. II, 733b 1-b739b 30.

25 El texto de la Theologia se puede encontrar en latín en Henry. Una selección de pasajes del texto se puede encontrar en inglés en Adamson The Arabic Plotinus. Las Enéadas se pueden encontrar traducidas al español por Jesús Igal en Gredos. 
tratado donde expone su visión de la filosofía aristotélica, a saber, su Falsafat aristûtâlî̀ ${ }^{26}$. No exploraremos las razones de este fenómeno; simplemente mencionaremos la tesis que aparece como más acertada y que sostiene que el filósofo árabe sospechaba que la Theologia era un texto apócrifo (LópezFarjeat, "La inmortalidad..." 600-1). Cuando al-Fârâbî menciona o cita la Theologia, es en aquellos textos donde argumenta en favor de la posibilidad de hacer funcionales las teorías cosmológicas y antropológicas de Platón y Aristóteles (Galston 13-4). De estas menciones, una en particular nos interesa para nuestro objetivo. En La armonía de las opiniones de los dos sabios: el divino Platón y Aristóteles, al-Fârâbî cita la Theología para intentar desenmarañar el problema del intelecto y la relación con el alma ${ }^{27}$ :

A veces al encontrarme a solas con mi alma, me desprendo de mi cuerpo y me convierto en sustancia abstracta e incorpórea. Entro en mi esencia, regreso a ella y me desprendo de cualquier otra cosa. Soy a la vez conocimiento, cognoscente y conocido. Veo en mi esencia tal belleza y esplendor que el asombro ante los mismos me aturde. En ese instante, me doy cuenta de que soy una mínima parte del mundo venerable y que a través de mi vida, actúo en él. Al estar seguro de esto, concedo que mi mente se eleve desde ese mundo hacia la divina causa y se funda con ella como si ambos fuésemos uno. Acto seguido, brillan hacia mí una luz y un esplendor tales que las lenguas no pueden describirlos ni los oídos escucharlos. Al encontrarme inmerso en aquella luz, alcanzar mi límite, y no poder soportarla más, desciendo al mundo del cálculo. El cálculo oculta de mí esa luz y, en ese momento, recuerdo cuando mi hermano Heráclito ordenaba buscar e indagar en el interior de la sustancia del alma venerable elevándose al mundo del intelecto. (Th.A IV.8 $)^{28}$

A partir de este texto podemos esclarecer un poco más el asunto. Comencemos por analizar el lenguaje. Si ponemos atención, los conceptos

\footnotetext{
26 En la bibliografía se menciona una traducción al inglés a cargo de Muhsin Mahdi.

27 Rashed disputa la autoría de este texto (19-58).

28 Seguimos aquí la traducción al español propuesta por López-Farjeat, "La inmortalidad..." 602.
} 
utilizados por el adaptador del texto (probablemente Porfirio, como mencionamos) tienen tintes más bien platónicos que aristotélicos. Se habla de desprenderse del cuerpo, de un mundo venerable, elevación y caída, etcétera. El lenguaje resuena al filósofo ateniense, a una visión dualista del cosmos y del ser humano. Vemos aquí un alma libre del cuerpo, capaz de transitar e iluminarse para alcanzar una existencia más allá del mundo; un alma, sin duda, capaz de alcanzar la inmortalidad ${ }^{29}$. Este pasaje es, quizás, el que expone de manera más evidente la influencia neoplatónica en al-Fârâbî, a través de la Theologia, y que le permite acercarse a una argumentación a favor de la inmortalidad del alma. Queda por explicar, sin embargo, cómo esa alma se relaciona con el cuerpo. Volvamos a los autores mencionados. Plotino sostiene que el alma puede existir separada del cuerpo, puesto que no se trata de un simple fenómeno que se da en un sujeto (Enn. IV.3, 20). De la misma forma, en la Theologia se refuta la idea del alma como existente a la manera de un predicado (no existente en sí mismo). Por el contrario, se afirma que la disolución del cuerpo no conlleva la corrupción del alma (Th.A II.91), puesto que se trata de una entelequia, no de una forma que cumpla la función de perfección de la materia (Th.A III.67-70). Plotino afirma algo similar y especifica el sentido en el que alma es entelequia (Enn. IV.7, 8.5). Este punto es de cierta relevancia, pues Plotino incorpora en su análisis el rol del intelecto agente. En el pasaje citado, el filósofo neoplatónico habla de "otra alma, la inteligencia". No queda duda de que, siguiendo a Alejandro de Afrodisias, se está refiriendo al intelecto agente, calificado como separable en el De anima. Sin embargo, como hemos mencionado, no queda del todo claro que para Aristóteles esa separabilidad fuese de carácter estricta. Pareciera ser que la interpretación plotiniana se aleja de la concepción que habría tenido el estagirita del intelecto agente pero que, neoplatonismo de por medio, esa interpretación exagerada o estricta es útil para los fines de Alejandro, Plotino y, finalmente, al-Fârâbî. Esta aproximación permite estructurar un argumento para sostener que el alma

29 Algunos han notado en este pasaje una mera intención de síntesis entre Platón y Aristóteles, más que una exposición seria, científica y precisa por parte de al-Fârâbî. Para esta aproximación ver Druart 134. 
humana, bajo el modelo jerárquico-emanacionista farabiano, y gracias al hecho de que el intelecto agente se entiende como un principio metafísico externo al ser humano, logra resistir la corrupción del cuerpo y es capaz de alcanzar la inmortalidad. Sin embargo, esta interesante concepción presenta un importante problema. López-Farjeat observa que la relación de cuerpo y alma expuesta en estos pasajes es expuesta de esta manera en los textos neoplatónicos y especialmente en la Theologia, pero no en el De anima. Esto implica que, de quererse argumentar en favor de la inmortalidad del alma, no hay fundamentos suficientes en el De anima (López-Farjeat, "La inmortalidad..." 596). Boeri sostiene una visión similar, señalando que la inmortalidad del alma es insostenible en el texto psicológico ${ }^{30}$.

Ya que la separabilidad del intelecto conduce a la inmortalidad del alma, revisemos brevemente el camino propuesto por al-Fârâbî para alcanzarla. Al parecer, se requiere que un cierto camino sea recorrido por el ser humano para alcanzarla, no siendo algo inmediato y gratuito para todos. Este camino es descrito en La ciudad ideal. Siguiendo la línea aristotélica, al-Fârâbî une la ética con la política y especifica las condiciones para lograr la felicidad que es, en resumen, la inmortalidad del alma:

Con la posesión de los primeros inteligibles posee el hombre tan solo una perfección primitiva. Tan solo esos inteligibles lo capacitarán para llegar a su última perfección. En esta consiste su felicidad, la cual se reduce a que el alma humana llegue a un modo de ser tan perfecto que ya para subsistir no necesite de la materia. (La ciudad 71)

Es muy interesante ver que en la búsqueda de la felicidad resuena claramente el platonismo: la posesión de los primeros inteligibles por la razón son los que abren el camino de la perfección. Es a través del conocimiento de ellos que el alma se prepara para alcanzar su mayor grado de elevación. Distingue, además, entre una perfección primitiva y una última perfección,

$30 \quad$ Para esta postura se recomienda revisar su interesante ensayo sobre la posibilidad de la inmortalidad del alma en los estudios introductorios a su traducción del De Anima. 
dejando entrever que la verdadera perfección no se encuentra en el plano sublunar. La perfección última solo se alcanza mediante un ejercicio cognoscitivo de acercamiento a las realidades suprasensibles. La última frase del pasaje es abiertamente platónica: la felicidad del ser humano consiste en lograr separar el alma del cuerpo y que ella pueda subsistir sin necesidad del cuerpo.

Sin embargo, líneas más adelante, al-Fârâbî se torna nítidamente aristotélico para describir ese camino de consecución de la felicidad:

Esta felicidad se llega a poseer mediante actos voluntarios, algunos de los cuales serán espirituales, mientras que otros serán corpóreos. No son cualesquiera actos, sino solamente ciertas acciones determinadas y concretas debido a disposiciones y hábitos interiores determinados y concretos [...] Es este un bien que se busca por sí mismo. No se busca para que, teniéndolo por cierto tiempo, mediante ese bien obtengamos otros ni después de la felicidad puede el hombre obtener otro bien mayor. Las acciones voluntarias que sirven para obtener la felicidad, son acciones honestas. Las disposiciones y hábitos interiores que han de proceder de tales acciones son las virtudes [...] Las acciones que impiden la felicidad son acciones malas y deshonestas. Las disposiciones y hábitos interiores de donde proceden esas acciones, son hábitos defectuosos y viciosos y viles. (72)

Este pasaje es un excelente y bello resumen de la ética aristotélica. Actos voluntarios, disposiciones interiores, hábitos, vicios, virtudes, una cierta concepción del fin último o bien supremo; todo resuena a los requisitos de Aristóteles para la vida buena y feliz. Así, junto con las condiciones externas necesarias (jerarquía de principios, intelecto agente, etcétera), al-Fârâbî detalla los requisitos internos para alcanzar la felicidad, que consiste en un ejercicio personal e interno de acciones buenas y nobles que se contraponen a acciones viles y viciosas. Estos dos pasajes, casi seguidos, representan la impronta de la filosofía de al-Fârâbî, a saber, un aristotelismo cruzado por nociones neoplatónicas, y adaptadas a la realidad histórica y religiosa de su tiempo. 
En el primer pasaje citado de La ciudad ideal, como mencionamos, hay un claro eco platónico: lo que permite la perfección al ser humano es el conocimiento de ciertas realidades suprasensibles y, partir de allí, perseguir su felicidad, que no es otra cosa que la separación de la materia, es decir, la subsistencia del alma. Este camino de ascenso epistemológico está marcado por ese intelecto agente, incorpóreo e inmortal. Es ese intelecto agente separado del mundo sublunar ${ }^{31}$ el que abre la posibilidad del conocimiento. Así, aunque manteniendo la mayoría de las cualidades del intelecto agente aristotélico del libro III del De anima (divino, incorpóreo, inmortal, eterno, etcétera), al-Fârâbî entiende la separabilidad de una forma drásticamente diferente. Es decir, toma lo que Aristóteles comprendió como una facultad, le agrega categorías cosmológicas neoplatónicas para ofrecer una salida a la aporía de la inmortalidad del alma del estagirita.

Retomemos el segundo pasaje. al-Fârâbî comienza haciendo una distinción aristotélicamente correcta, a saber, hay actos que son voluntarios y otros que son involuntarios. En general los actos voluntarios se asocian al uso de la razón y la voluntad, mientras que los irracionales están asociados a los instintos ${ }^{32}$. A su vez, algunos de estos actos serán espirituales, como el pensamiento, y otros corpóreos, como el hambre o el frío. El correcto ordenamiento de esos actos, configurados como hábitos buenos permiten alcanzar la felicidad, que es un bien en sí mismo, fuera del cual no se necesita o anhela nada más. Cuando el ordenamiento es hacia el bien, esa disposición recibe el nombre de virtud, y cuando se dirige al mal y aleja de la felicidad corresponde a actitudes viciosas. Sin embargo, en la antropología aristotélica, todo esto es posible por la posesión del intelecto como facultad, no como principio externo. Es llamativo que al-Fârâbî escinde el intelecto agente de la capacidad racional. Es como si el ser humano no fuera per se racional, sino que adquiere esta cualidad solo por el efecto externo del intelecto agente. Con ello, aunque recogiendo formalmente su ética, el árabe se desmarca de la doctrina de las facultades superiores de Aristóteles y se acerca a una

\footnotetext{
31 De acuerdo con el apartado anterior, Aristóteles habría pensado la separabilidad bajo otras categorías y no como la concibió la filosofía árabe clásica.

32 Esto podría presentarse con ciertos matices que no son relevantes para nuestro objetivo.
} 
cosmología con tintes más bien platónicos. Este último aspecto retrata de forma nítida la filosofía de al-Fârâbî: una filosofía aristotélica mezclada con el platonismo heredado de Alejandro y Plotino, llevada a cabo de tal forma que pareciera no entrañar contradicciones internas, pero que, claramente, incorpora elementos externos a las filosofías de Platón y Aristóteles ${ }^{33}$.

\section{Conclusión}

En este texto se ha hecho un esfuerzo por mostrar algunas ideas centrales. Primero, que el problema de la inmortalidad del alma, en el estricto marco de la filosofía aristotélica expuesta en el De anima, constituye una aporía. Segundo, que el abordaje del problema que desarrolla al-Fârâbî, aunque concebido bajo las categorías y la psicología de Aristóteles, incluye elementos que exceden lo expuesto por el filósofo y termina ofreciendo una solución que claramente incorpora elementos neoplatónicos, no logrando resolver la aporía de la inmortalidad en los términos del estagirita. En este sentido, la teoría farabiana de la inmortalidad consiste en una mezcla entre los ambiguos pasajes del De anima, la lectura que Alejandro de Afrodisias hace de ellos, el texto apócrifo de la Theologia y la jerarquía de los principios de al-Fârâbî, no resolviendo las interrogantes planteadas por Aristóteles, sino superándolas en la incorporación de otros sistemas de pensamiento. Finalmente, se intentó mostrar cómo la propuesta del filósofo para alcanzar la felicidad (inmortalidad) es una perfecta muestra de su pensamiento: un aristotelismo neoplatonizado que busca resolver aquellos aspectos que la filosofía peripatética no logró zanjar de manera definitiva llevando a cabo una síntesis entre Platón y Aristóteles.

En un futuro trabajo sería interesante ahondar en cómo esta y

33 al-Fârâbî dedica una obra a presentar de forma sistemática la filosofía de estos dos maestros (Kitâb al-Jam' bayn Ra'yay al-Hakîmayn Aflâtûn al-Ilâhî wa Aristûtâlîs). Allí se puede evidenciar la recepción que lleva a cabo de cada uno en distintas disciplinas, con una refinación mayor que los pasajes que hemos citado, pero que no logran ilustrar la síntesis que desarrolla de ambos. En la bibliografía se menciona una traducción al inglés a cargo de Butterworth y otra al español de Guerrero. 
otras teorías de la inmortalidad le permitieron a los filósofos medievales, especialmente a Santo Tomás de Aquino, proponer su propio acercamiento al problema, ya no desde el neoplatonismo, sino en el marco del mundo cristiano occidental, y de la misma forma, cómo esa aproximación influyó en otros períodos en los que se recoge al mundo clásico como, por ejemplo, la Ilustración. 


\section{Bibliografía}

Adamson, Peter. The Arabic Plotinus. Londres: Duckworth, 2002.

_. "The Theology of Aristotle". Standford Encyclopedia of Philosophy, junio 2008, www.plato.stanford.edu/entries/theology-aristotle/

al-Fârâbî. La ciudad ideal (Mabâdi' ârâ' abl al-madînah al-fâdilah). Traducido por Manuel Alonso. Madrid: Tecnos, 2002.

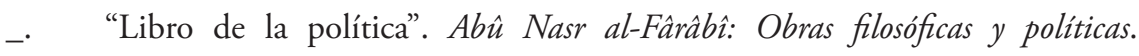
Traducido por Rafael Guerrero. Madrid: Trotta, 2008.

_. On the Perfect State (Mabâdi' ârấ abl al-madînah al-fâdilah). Traducido por Richard Walzer. Oxford: Oxford UP, 1985.

_. "The Harmonization of the Two Opinions of the Two Sages: Plato the Divine and Aristotle" (Kitâb al-Jam'bayn Ràjay al-Hakimayn Aflâtûn al-Ilâhî̀ wa Aristuttallis). Alfarabi: The Political Writings. Selected Aphorisms and Other Texts. Traducido por Charles Butterworth. Londres: Cornell UP, 2001.

_. "The Philosophy of Aristotle" (Falsafat Aristûtâlîs). Alfarabis Philosophy of Plato and Aristotle. Traducido por Muhsin Mahdi. Nueva York: The Free Press of Glencoe, 1969.

Aristóteles. Metafísica. Traducido por Valentín García Yebra. Madrid: Gredos, 2018.

Aristóteles. Acerca del alma. Traducido por Marcelo Boeri. Buenos Aires: Colihue, 2010.

Aristóteles. Reproducción de los animales. Traducido por Ester Sánchez. Madrid: Gredos, 1994.

Badawi, A. Plotinus apud Arabes. Cairo: Dirasa Islamiyya, 1947.

Boeri, Marcelo. "Introducción”. Aristóteles. Acerca del alma. Buenos Aires: Colihue, 2010 .

Cruz Hernández, Miguel. "La teología del pseudo-Aristóteles (Kitâb Utûlûŷ̂ya LiAristû) y la estructuración del neoplatonismo islámico". Anuario Filosófico, n. ${ }^{\circ} 33,2000$, pp. 87-110.

Di Camillo, Silvana. "Aristóteles frente a Platón en torno a la separación y eternidad de la forma". Páginas de Filosofía, año 18, n. ${ }^{\circ} 21,2017$, pp. 140-63. 
Dieterici, Friedrich. Alfârâbî̀s philosophische Abhandlungen. Leiden: Brill, 1890.

Druart, Therese-Anne. "Al-Fârâbî, Emanation and Metaphysics". Neoplatonism and Islamic Thought. Nueva York: State University of New York Press, 1992, 12748.

Galston, Miriam. "A Re-examination of al-Fârâbî's Neoplatonism”. Journal of the History of Philosophy, n. ${ }^{\circ}$ 15, 1977, pp. 13-32.

Gilson, Étienne. La filosofía en la Edad Media. Madrid: Gredos, 1965.

_. $\quad$ "Les sources gréco-arabes de l'augustinisme avicennisant". Archives d'Historie Doctrinale et Litteraire du Moyen Age, n. ${ }^{\circ} 4$, 1929, pp. 5-149.

Guerrero, Rafael. La recepción árabe del De Anima de Aristóteles: Al-Kindi y AlFarabi. Madrid: Consejo Superior de Investigaciones Científicas, 1992.

Henry, P. y Schwyzer, H. R. Plotini Opera, vol. 2. París: Desclée de Brouwer, 1959.

Jaeger, Werner. Aristóteles. Bases para la historia de su desarrollo intelectual. México: FCE, 1946.

López-Farjeat, Luis Xavier. "Cosmología, biología y origen del alma en al-Fârâbî y Avicena”. Ideas y Valores, vol. 68, n. ${ }^{\circ} 119$, 2019, pp. 13-32.

_. $\quad$ "La inmortalidad del alma en la Theologia pseudoaristotélica y su papel en la filosofía de al-Fârâbî”. Estudios de Asia y África, vol. 40, n. ${ }^{\circ}$ 3, 2005, pp. 577606.

Mahdi, Mushin. "The Editio Princeps of Fârâbî̀s Compendium Legum Platonis". Journal of Near Eastern Studies, vol. 20, n. ${ }^{\circ}$ 1, 1961, pp. 1-24.

Minio-Paluello, Lorenzo. Opuscula. The Latin Aristotle. Ámsterdam: Hakkert, 1972.

Nuyens, François. L'évolution de la Psychologie D'Aristote. Lovaina: Éditions de l'Institut Supérieure de Philosophie, 1948.

Peters, Francis Edwards. Aristoteles Arabus. The Oriental Translations and Commentaries on the Aristotelian Corpus. Leiden: Brill, 1968.

Plotino. Enéadas III-IV. Traducido por Jesús Igal. Madrid: Gredos, 1985.

_. $\quad$ Enéadas V-VI. Traducido por Jesús Igal. Madrid: Gredos, 1998.

Rashed, Marwan. "Al-Farabi's Lost Treatise on Changing Beings and the Possibility of a Demonstration of the Eternity of the World". Arabic Sciences and Philosophy, vol. 18, n. ${ }^{\circ} 1,2008$, pp. 19-58. 
Rosenthal, Franz. "On the Knowledge of Plato's Philosophy in the Islamic World". Islamic Culture, n. ${ }^{\circ} 14,1990$, pp. 387-422.

Thillet, Pierre. "Sagesse grecque et philosophie musulmane". Le mardis de Dar elSalam. Paris: J. Vrin, 1958, pp. 55-93.

Zingano, Marco. Razão e Sensação em Aristóteles. Puerto Alegre: LPM, 1998.

Zimmermann, Fritz W. "The Origins of the so-called Theology of Aristotle". Warburg Institute Surveys and Texts XI: Pseudo-Aristotle in the Middle Ages. Londres: Warburg Institute, 1986, pp. 110-240. 